\title{
PATHOPHYSIOLOGY \& MANAGEMENT OF ISCHEMIC AND NEUROPATHIC PAIN
}

\author{
Sadaf Ahmed ${ }^{1 \& 2}$ \& Shamoon Noushad ${ }^{1}$ \\ 1. Advance Educational Institute \& Research Centre (AEIRC) \\ 2. University of Karachi \\ Corresponding Author Email: sadafivy@ gmail.com
}

\begin{abstract}
Both nociceptive and neuropathic types of pain utilize the same nervous pathway for conduction with considerable physiologic disparities exist in the mechanism through which the body processes and resolves these painful stimuli. Nociceptive pain that results from a known or obvious source (e.g. trauma, cancer metastasis, ischemia, arthritis) is often simple to recognize. Neuropathic pain, however, may occur with the nonappearance of any particular precipitating cause. Anticonvulsants, topical anesthetic agents, antidepressants, antiarrhythmic, anti-inflammatory agents, opioid analgesics, NMDA receptor antagonists, combined analgesic therapy, physiotherapeutic modalities and other ways are used as treatment. Other interventions such as motor cortex stimulation, transcutaneous electrical nerve (TENS) stimulation units, and other peripheral stimulation have been shown to be supportive. A frequent sequella of both types of diabetes is the development of peripheral neuropathy in either motor or sensory nerves, or both. Highfrequency TENS can suppress abnormal, spontaneous activity in a damaged peripheral nerve and can block nociceptive transmission in the dorsal horn, the use of this practice seems a practical approach for relief of neuropathic pain. Indeed, TENS applied to the lower extremities of neuropathic pain sufferers reduces the intensity of pain. However what actual molecular mechanism is followed is still a mystery and these studies emphasize for carrying out further work to correlate the neuropathic and ischemic aspects of disorders in these patients.
\end{abstract}

\section{KEYWORDS}

Neuropathic pain, Transcutaneous electrical nerve stimulation, Ischemic pain, Nociception, Diabetic peripheral neuropathy

\section{INTRODUCTION}

The nociception or neuropathy can be a foundation of importunate pain, involve similar neuronal pathways but considerable physiological differences. Nociceptive pain that results from a known or obvious source from stimulus to response and from pain to resolution (Merskey, 1994; Dworkin, 2002). Examples of the former include diabetic peripheral neuropathy (DPN), postherapeutic neuralgia (PHN), antineoplastic therapy sensory neuropathy, reflex sympathetic dystrophy, trigeminal neuralgia along with deafferentation syndromes resulting in neuropathic pain include central post stroke pain, Parkinson disease etc.

The neuropathic pain tends to show a moderately reduced response to conventional pain reliever (Chen, 2004; Namaka, 2004). There is no actual restoration to health after neuropathy; yet, to make it less severe or intense and enhancement on the whole in eminence of healthy being are the core objectives of management. Anticonvulsants, anesthetic agents, antidepressants, antiarrhythmic, antiinflammatory agents, opioid analgesics, NMDA receptor antagonists, combined analgesic therapy, physiotherapeutic modalities and other ways are used as treatment. Though much of the assessments and managements resulted in non significant results without any considerable change in patients sufferings, such cases must referred to pain clinics. Optional intrusion such as motor cortex stimulation (Brown, 2005) transcutaneous electrical nerve stimulation (TENS) units, and other peripheral stimulations (Johnson, 2004) have been helpful in many uncontrollable cases. High-frequency TENS can restrain irregular, impulsive activity in an injured peripheral nerve and can block nociceptive transmission in the dorsal horn, the use of this modality seems a viable treatment for the pain of diabetic neuropathic pain. Indeed, TENS applied to the lower extremities of patients with diabetic neuropathy reduces the intensity of pain experienced in the distal portion of the same limb. A frequent sequella of both types of diabetes is the development of peripheral neuropathy in either motor or sensory nerves, or both a consequence of any neuropathy affecting motor and sensory peripheral nerves is reduction or loss of strength and sensation. Another potential consequence of peripheral neuropathy in people with diabetes is severe, unremitting pain people with painful diabetic neuropathy describe their pain as constant burning or blazing (Watkins, 1990).

Pathway by which painful peripheral neuropathy occurs in people with diabetes is not well-understood; however, abnormal activation of damaged or regenerating peripheral nerves may be involved as these nerves become upset at intraneuronal levels due to abnormal metabolic pathway for the carbohydrate (Horowitz,1993; Schoemaker,1993) or when blood supply to the nerve becomes perturbed because of perineural vascular changes (Horowitz,1993) Although all nerve fibers may be injured by these processes, small myelinated and unmyelinated fibers that carry out pain information are most affected once damaged, the axons of these small-diameter nerve fibers undergo regeneration (Brown,1976) and pain may occur through abnormal activation of damaged or regenerating small-diameter nociceptive fibers (Portenoy, 1989). Thus, the perception of this pain is dependent on neurotransmission in the dorsal horn of the spinal cord. Given this etiology for the pain of diabetic neuropathy, a possible management would hinder 
the anomalous activity in the pretentious peripheral nerve or blocked neurotransmission. Transcutaneous or straight stimulation of sensory peripheral nerves is believed to create analgesia through both of these mechanisms.

Patients with peripheral arterial disease (PAD) may develop a broad range of peripheral nerve dysfunctions including pain and sensory deficiencies due to chronic ischemia mostly involving the lower limbs (Lang, 2006). To investigate the degree of sensory abnormalities in such patients quantitative sensory testing (QST) might be a useful tool. Pain is induced by injury or disease of the nervous system. Any local nerve injury tends to spread to distant parts of the peripheral and central nervous system. In particular, the efficacy of opioids at the spinal level is much decreased following nerve injury. Repeated or prolonged noxious stimulation and the persistent abnormal input following nerve injury activate a number of intracellular second messenger systems, implying phosphorylation by protein kinases, particularly protein kinase $\mathrm{C}$ (PKC). Intracellular signal cascades result in immediate early gene (IEG) induction which is considered as the overture of a extensive change in protein synthesis, this progression of escalating nervous excitability may be measured as a strategy to recompense practical scarcity subsequent nerve injury. There is an important contribution of tissue ischemia to pain. It has been suggested that endothelial cell production, known to be induced by cytokines produced by macrophages, indicates increased action and pathogenic implication of these cytokines in the pain model (Sommer, 1996). Furthermore, endothelial dysfunction in type 2 diabetics mellitus with neuropathy has been reported (De Angelis, 2001).

\section{NEUROPATHIC PAIN, ISCHEMIA AND ELECTRICAL STIMULATION}

Considering characteristics that nociceptive and neuropathic pain have in common will help clarify their differences. Pain signals resulting from noxious stimuli (wounds, thermal or inflammatory insults) are converted into electrical impulses within tissue nociceptors whose cell bodies are found in dorsal root ganglions; both nociceptive and neuropathic pain signals utilize the same pain pathways. Information regarding intensity, quality, and location of pain is conveyed to the sensory cortex from the somatosensory thalamus. The central nervous system utilizes descending inhibitory pathways via the dorsolateral fasciculus (Lissauer's tract) of the spinal cord and the periaqueductal gray matter to modulate transmission of nociceptive stimuli. Complex equilibrium of painsignaling and pain-relieving pathways connecting peripheral and central nervous systems. Efficient, rapid transmission of acute responses to a painful stimulus is a self-protection process can be characterized. Pain signals an "alarm" that leads to subsequent protective responses. Neuropathic pain, however, signals no imminent danger. The operative difference is that neuropathic pain represents a delayed, ongoing response to damage that is no longer acute which continues to be expressed as painful sensations. Sensory neurons damaged by injury, disease, or drugs produce spontaneous discharges that lead to sustained levels of excitability. These ectopic discharges begin to "cross talk" with adjacent uninjured nerve fibers, resulting in amplification of the pain impulse (peripheral sensitization).

The hyper excitability leads to increased transmitter release causing increased response by spinal cord neurons (central sensitization). The process, known as "windup," accounts for the fact that the level of perceived pain is far greater than what is expected based on what can be observed (Ji, 2004). Painful nerve stimulation leads to activation of N-methyl-Daspartate (NMDA) receptors on the postsynaptic membrane in the dorsal horn of the spinal cord. Release of NMDA, a modulating neurotransmitter, is coupled with subsequent release of glutamate, an excitatory neurotransmitter. The resultant extended depolarization (influx of calcium and sodium and efflux of potassium) produces much larger than usual postsynaptic potentials, known as synaptic potentiation. Spinal windup has been continuous increased excitability of central neuronal membranes with persistent potentiation" (Spruce, 2003). Neurons of the peripheral and central nervous system continue to transmit pain signals beyond the original injury, thus activating an ongoing, unremitting pain response. Devor (1993) presented evidence showing that damaged sensory fibers have a higher concentration of $\mathrm{Na}^{+}$ channels, an alteration that would augment spontaneous firing. The symptoms described by persons with neuropathic pain are myriad, representing a variety of possible nerve injuries implicated in the etiology. Neuropathic pain sufferers complain of numbness, burning, or tingling, or a combination; they describe prickly or pins and needles sensations. Boureau (1990) identified six adjectives used substantially more frequently to describe neuropathic pain. The stimulating shock, burning, and tingling were most commonly used, along with cold, pricking, and itching. These expressions are suggestive of a neuropathic etiology for pain. Several common types of responses are elicited from patients with neuropathic pain. These abnormal sensations, or dysesthesias alone, or in combination with specific complaints. dissimilar to the common responses to nociceptive pain, the exasperating or painful feelings occurs completely in the absence of an obvious source as severe aching by a chilly flow of air on the cheek of a patient suffering from trigeminal neuralgia. Allodynia is the term given to a painful response to an otherwise benign stimulus. Taken to the extreme this can result in the unbearable neuropathic symptom known as hyperpathia. Another example of allodynia is touch sensitivity of badly sunburned skin, so even slight hitting of the inflamed area causes extreme discomfort; like neuropathic pain, this reaction turns out of fraction to the intensity of injury. The discomfort of one's foot "falling asleep" is an ordinary paresthesia a kind of sore sensation that can be resolved unexpectedly, contrasting from constant, self-perpetuating and annoying sensation of pins and needles caused by neuropathic pain. When the sciatic nerve is sectioned in the upper leg of rats, a neuroma is induced in the distal stump of the cut nerve, spontaneous activity develops in the neuroma. This spontaneous activity is believed to cause pain because it occurs in small-diameter, flippantly myelinated peripheral 
nerve fibers, and known to transmit painful information (Collins, 1960). Even a 6-second direct stimulation $(100 \mathrm{~Hz})$ of the sciatic nerve proximal to the neuroma lowers abnormal, impulsive as much as an hour after the stimulation(Wall, 1974). Peripheral electrical stimulation can also block transmission of nociceptive impulses in the dorsal horn of the spinal cord. It is not known whether TENS would be as effective if it were delivered through electrodes placed on the skin of the low back that is an option for curing pain in the lower extremities. This electrode placement delivers therapeutic stimulation through non pathological nerves (dorsal rami) to many of the same spinal cord segments that innervate the lower extremity. For several reasons, such an alternative electrode placement might be desirable when TENS is used to treat people with painful diabetic neuropathy (Sadaf, 2013). The myelinated thick nerve fibers may be damaged in the painful extremities of people with severe diabetic neuropathy. Because activation of large-diameter myelinated nerve fibers is believed to be the mechanism by which high-frequency TENS produces analgesia, (Wall, 1974; Garrison, 1994 \& Wall, 1967) the modality may be more effective for people with severe neuropathy if it is delivered through undamaged peripheral nerves. Another reason to consider not placing the electrodes on the painful extremity is the concern for integument integrity. Pattern of decreased perception suggests deafferentation of multiple fiber contribution, the occurrence of allodynia suggests that central sensitization also plays a role in the pain state of peripheral arterial disease patients. Subgroup analysis points towards a disease associated peripheral neuropathy independent of diabetes (Lang, 2006). The behavioral parameters have been exploited to study the pharmacology and modulation of neuropathic pain at any abnormal ectopic excitability by Nerve fibers develop near the site of nerve injury. That can be unusual allocation of $\mathrm{Na}(+)$ channels, as well as abnormal responses to endogenous pain producing substances and cytokines such as tumor necrosis factor alpha (TNF-alpha). Persistent abnormal excitability of sensory nerve endings in a neuroma is considered a mechanism of stump pain after amputation (Zimmermann, 2001).

\section{CONCLUSION}

There are variety of ways to treat patients with neuropathic pain. However, no sure cure for neuropathic pain disorders exists, and it is essential to search for new ways and approaches. Hence, we propose that electro stimulation may bring better therapeutic management of patients with neuropathic pain. Effect of blood supply and ischemia on the neuronal structures in human disorders is evident. On the other hand, the disordered conditions and processes involved in peripheral neuropathic pain have also been extensively studied. However, we have yet to know the significance of vascular and endothelial activities in understanding neuropathic pain. The present view is to carry out neurovascular background of exploring the mechanisms involved in neuropathic pain. Pathways by which painful peripheral neuropathy occurs in people with diabetes is not well- understood. However, the cellular pathobiology including apoptosis suggests future strategies against neuropathic pain that emphasize preventive aspects.

\section{REFERENCES}

- Boureau, F., Doubrere, J. F., \& Luu, M. (1990). Study of verbal description in neuropathic pain. Pain, 42(2), 145152.

- Brown, J. A., \& Pilitsis, J. G. (2005). Motor cortex stimulation for central and neuropathic facial pain: a prospective study of 10 patients and observations of enhanced sensory and motor function during stimulation. Neurosurgery, 56(2), 290-297.

- Brown, M. J., Martin, J. R., \& Asbury, A. K. (1976). Painful diabetic neuropathy: a morphometric study. Archives of neurology, 33(3), 164-171.

- Camargo, F. D., Green, R., Capetenaki, Y., Jackson, K. A., \& Goodell, M. A. (2003). Single hematopoietic stem cells generate skeletal muscle through myeloid intermediates. Nature medicine, 9(12), 1520-1527.

- Chen, H., Lamer, T. J., Rho, R. H., Marshall, K. A., Sitzman, B. T., Ghazi, S. M., \& Brewer, R. P. (2004). Contemporary management of neuropathic pain for the primary care physician. In Mayo Clinic Proceedings (Vol. 79, No. 12, pp. 1533-1545). Elsevier.

- Coderre, T. J., Xanthos, D. N., Francis, L., \& Bennett, G. J. (2004). Chronic post-ischemia pain (CPIP): a novel animal model of complex regional pain syndrome-type I (CRPS-I; reflex sympathetic dystrophy) produced by prolonged hindpaw ischemia and reperfusion in the rat. Pain, 112(1), 94-105.

- Collins Jr, W. F., Nulsen, F. E., \& Randt, C. T. (1960). Relation of peripheral nerve fiber size and sensation in man. Archives of neurology, 3(4), 381.

- De Angelis, L., Marfella, M. A., Siniscalchi, M., Marino, L., Nappo, F., Giugliano, F., \& Giugliano, D. (2001). Erectile and endothelial dysfunction in Type II diabetes: a possible link. Diabetologia, 44(9), 1155-1160.

- Devor, M., Govrin-Lippmann, R., \& Angelides, K. (1993). $\mathrm{Na}+$ channel immunolocalization in peripheral mammalian axons and changes following nerve injury and neuroma formation. The Journal of neuroscience, 13(5), 1976-1992.

- Dworkin, R. H. (2002). An overview of neuropathic pain: syndromes, symptoms, signs, and several mechanisms. The Clinical journal of pain, 18(6), 343-349.

- Garrison, D. W., \& Foreman, R. D. (1994). Decreased activity of spontaneous and noxiously evoked dorsal horn cells during transcutaneous electrical nerve stimulation (TENS). Pain, 58(3), 309-315.

- Horowitz, S. H. (1993). Diabetic neuropathy. Clinical orthopaedics and related research, 296, 78-85.

- Ji, R. R., \& Strichartz, G. (2004). Cell signaling and the genesis of neuropathic pain. Science Signaling, 2004(252), re14.

- Johnson, M. D., \& Burchiel, K. J. (2004). Peripheral stimulation for treatment of trigeminal postherpetic neuralgia and trigeminal posttraumatic neuropathic pain: a pilot study. Neurosurgery, 55(1), 135-142. 
- Lang, P. M., Schober, G. M., Rolke, R., Wagner, S., Hilge, R., Offenbacher, M., Treede, R. D., Hoffmann, U., Irnich, D. (2006). Sensory neuropathy and signs of central sensitization in patients with peripheral arterial disease.Pain, 124(1-2), 190-200.

- Lee, K. H., Chung, J. M., \& Willis Jr, W. D. (1985). Inhibition of primate spinothalamic tract cells by TENS. Journal of Neurosurgery, 62(2), 276-287.

- Merskey, H., \& Bogduk, N. Task Force on Taxonomy of the International Association for the Study of Pain. Classification of chronic pain: descriptions of chronic pain syndromes and definition of pain terms. Seattle: IASP, 1994. ISBN 978-0-931092-05-3.

- Namaka, M., Gramlich, C. R., Ruhlen, D., Melanson, M., Sutton, I., \& Major, J. (2004). A treatment algorithm for neuropathic pain. Clinical therapeutics, 26(7), 951-979.

- Portenoy, R. K. (1989). Painful polyneuropathy. Neurol Clin., 7, 265-288

- Sadaf, A., Hira, Z., Shamoon, N., \& Lubna, A. (2013). Role of Tens Therapy in Neuropathic Pain. International Journal of Pharmaceutical \& Biological Archives. 4(6), $1175-1180$.

- Schoemaker, J. H. (1993). Pharmacological treatment of diabetic peripheral neuropathy: challenges and possibilities. The British journal of clinical practice, 48(2), 91-96.

- Shi, Q., Rafii, S., Hong-De Wu, M., Wijelath, E. S., Yu, C., Ishida, A., \& Hammond, W. P. (1998). Evidence for circulating bone marrow-derived endothelial cells.Blood, 92(2), 362-367.

- Sommer, C., \& Myers, R. R. (1996). Vascular pathology in $\mathrm{CCl}$ neuropathy: A quantitative temporal study. Experimental neurology, 141(1), 113-119.

- Spruce, M. C., Potter, J., \& Coppini, D. V. (2003). The pathogenesis and management of painful diabetic neuropathy: a review. Diabetic Medicine, 20(2), 88-98.

- Suuronen, E. J., Wong, S., Kapila, V., Waghray, G., Whitman, S. C., Mesana, T. G., \& Ruel, M. (2006). Generation of CD133+ cells from CD133- peripheral blood mononuclear cells and their properties. Cardiovascular research, 70(1), 126-135.

- Urbich, C., Heeschen, C., Aicher, A., Dernbach, E., Zeiher, A. M., \& Dimmeler, S. (2003). Relevance of monocytic features for neovascularization capacity of circulating endothelial progenitor cells. Circulation, 108(20), 2511-2516.

- Wall, P. D. (1974). Properties of afferent nerve impulses originating from a neuroma. Nature, 248, 740-743.

- Wall, P. D., \& Sweet, W. H. (1967). Temporary abolition of pain in man. Science, 155(3758), 108-109.

- Watkins, P. J. (1990). Natural history of the diabetic neuropathies. The Quarterly journal of medicine, 77(284), 1209-1218.

- Zimmermann, M. (2001). Pathobiology of neuropathic pain. European journal of pharmacology, 429(1), 23-37. 